\section{High Birefringence Fibre Interferometer for Optical Sensing Applications}

\author{
D. C. C. Norman, Y. Lai and D. J. Webb
}

We describe the use of high birefringence fibre forming a differential path interferometer for heterodyne fibre optic sensing applications.

Introduction: Interferometric techniques in optical fibre sensor applications offer extremely high sensitivity measurements that allow the development of high performance optical sensor schemes. The ability to generate a carrier signal by modulating interferometers with a $2 \pi$ serrodyne waveform has been successfully applied in many interferometric fibre optic sensor systems [1].

Common arrangements for two-beam interferometers include the Michelson and the Mach-Zehnder interferometer, both of which can be used to generate a beat frequency. Differential path interferometers are analogous to the Mach-Zehnder interferometers but with this technique the two beams are carried within a single fibre. High birefringence (Hi-Bi) fibre can be used to form a differential path interferometer by exploiting the fast and slow axis in the core of the Hi-Bi fibre [2].

In this letter we report on the use of a differential path interferometer formed in $\mathrm{Hi}-\mathrm{Bi}$ fibre modulated to create a carrier frequency and demonstrate its performance in two

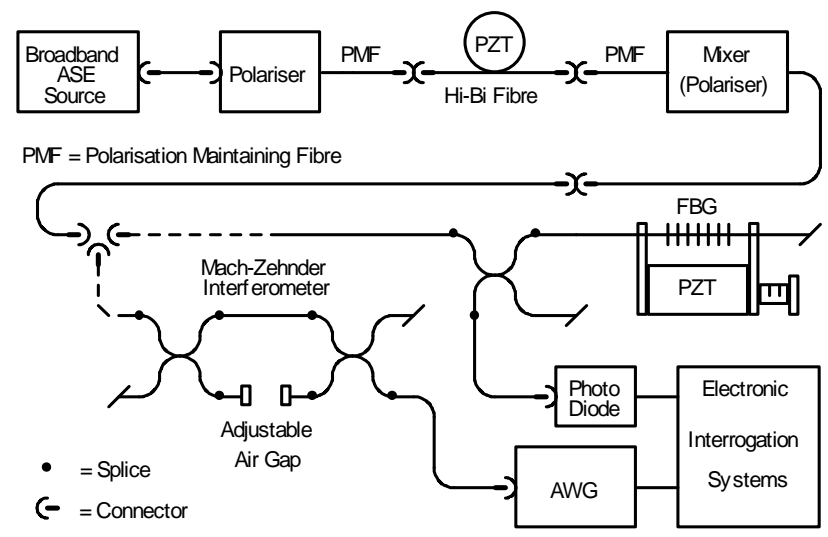

Fig. 1 Experimental set-up.

fibre optic sensor schemes. Firstly, fibre Bragg grating (FBG) sensor interrogation using a heterodyne approach adopting interferometric wavelength shift detection [3]. Secondly, interferometric sensor interrogation combining heterodyne processing and the dual wavelength approach [4].

Experimental Set-Up: The experimental set-up is shown in Fig. 1. A non-polarised erbium-doped fibre amplified spontaneous emission source provided a total output power of $12 \mathrm{dBm}$ over a spectral width of $33 \mathrm{~nm}$ centred on a wavelength of $1545 \mathrm{~nm}$. The light passed through a polariser, into a length of Polarisation maintaining fibre (PMF), through a rotating connector arrangement and into the Hi-Bi fibre wound around a piezoelectric fibre stretcher forming a differential path interferometer. The piezoelectric fibre stretcher was connected to a function generator able to produce a $2 \pi$ amplitude serrodyne waveform. The light then passed through a second rotating connector, through a mixer (second polariser) and into a selective connector directing the light to either the FBG or interferometric sensor. The FBG sensor consisted of a single Bragg grating mounted onto a second piezoelectric fibre stretcher so that dynamic strain signals could be applied, reflected light from the FBG sensor was directed through a coupler to a photodiode and relevant electronic interrogation system. The interferometric sensor consisted of a Mach-Zehnder interferometer with an air gap in one arm to adjust the optical path difference (OPD). The light output from the interferometer was connected to an arrayed waveguide grating (AWG) interrogation scheme $[5,6]$.

Hi-Bi Fibre Interferometer: Polarised light input into Hi-Bi fibre at an azimuth of 45 degrees with respect to the birefringence axis of the fibre will degenerate into two noninterfering orthogonal polarised modes which propagate within the core of the fibre with a differential delay determined by the birefringence, $\Delta \mathrm{n}$ and the length of the fibre, L. The detected light at the output of the Hi-Bi fibre after passing through a mixer exhibits periodic intensity variations against the input wavelength [2]. The free spectral range (FSR) of the periodic interferometer output is given by

$$
F S R=\frac{\lambda^{2}}{\Delta n L}
$$

Where, $\lambda$ denotes the central wavelength of the light source and $\Delta \mathrm{nL}$ is the OPD of the interferometer. A $21.56 \mathrm{~m}$ length of Hi-Bi fibre was selected to produce an FSR of $0.3 \mathrm{~nm}$ and an associated OPD of $8 \mathrm{~mm}$. The carrier signal was created by winding $20 \mathrm{~m}$ of $\mathrm{Hi}-\mathrm{Bi}$ fibre around a piezoelectric fibre stretcher and applying a ramp waveform sufficient in amplitude to drive the interferometer over a single interference fringe. We demonstrated this by increasing the amplitude of a $0.25 \mathrm{~Hz}$ ramp waveform and monitoring the fringe positions at the output of the Hi-Bi interferometer with an optical spectrum analyser. The differential nature of the Hi-Bi interferometer means that both arms are subjected to the modulation at differing degrees. This results in an increased amount of strain that we need to apply to the fibre to drive the interferometer over a fringe than that would be necessary if we were to use a two-beam interferometer such as a Mach-Zehnder. The strain we applied to drive the Hi-Bi interferometer over one fringe was approximately $6 \mu \varepsilon$. 
FBG Sensing: The first demonstration using the Hi-Bi fibre interferometer adopts a heterodyne approach using interferometric wavelength shift detection [3]. We

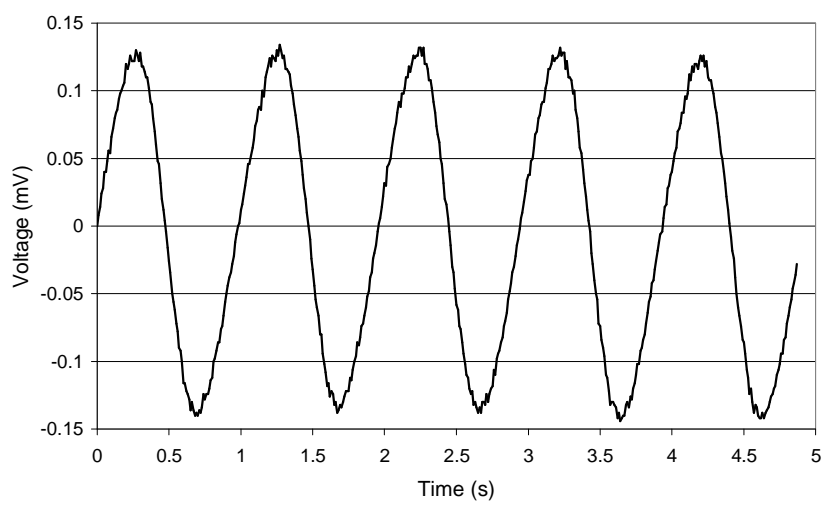

Fig. 2 Recovered $1 \mathrm{~Hz}$ strain signal.

modulated the phase of the $\mathrm{Hi}-\mathrm{Bi}$ interferometer at $20 \mathrm{~Hz}$ and applied a low frequency strain amplitude of $1 \mu \varepsilon$ at $1 \mathrm{~Hz}$ to the FBG sensor. The FBG had a centre wavelength of $1558.29 \mathrm{~nm}$ and a $-3 \mathrm{~dB}$ linewidth of $0.13 \mathrm{~nm}$. The output of the photodiode was bandpass filtered (at the carrier frequency) and connected to a lock-in amplifier (LIA). We used the serrodyne modulation frequency as the reference and monitored the phase shift using an oscilloscope connected to the output of the LIA. The results are shown in Fig. 2 and show the recovered $1 \mathrm{~Hz}$ strain signal applied to the FBG sensor.

Interferometric sensing: In this demonstration we used the $\mathrm{Hi}$-Bi fibre interferometer as a processing interferometer away from the Mach-Zehnder interferometer sensor with its

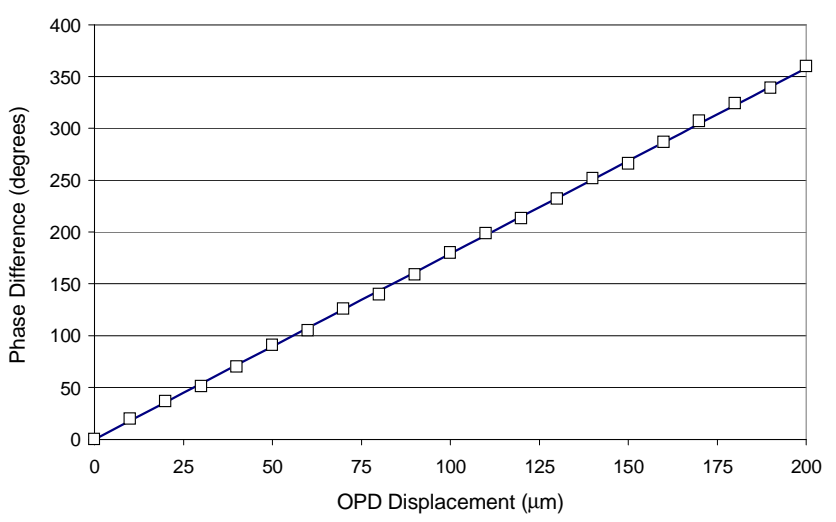

Fig. 3 Phase difference vs. Mach-Zehnder OPD.

Experimental results (squares). Theoretical relationship indicated by the solid line.

OPD closely matched to the OPD of the processing interferometer forming a composite, coherence tuned system [7]. We again modulated the phase of the Hi-Bi interferometer at $20 \mathrm{~Hz}$ and used an AWG to synthesise two separate low coherent light sources illuminating the system [6]. We then monitored their phase difference using a LIA as we adjusted the OPD of the Mach-Zehnder interferometric sensor. The results are shown in Fig. 3. The unambiguous range in terms of a change in the Mach-Zehnder OPD is $200 \mu \mathrm{m}$ for the two selected synthesised light sources. The errors in the experimental values are predominantly due to the Hi-Bi and Mach-Zehnder interferometers, which are prone to fluctuations due to draughts or temperature changes in the laboratory.

Conclusion: The experimental work reported has demonstrated that an interferometer formed in $\mathrm{Hi}$-Bi fibre can be modulated using a piezoelectric stretcher to produce a low frequency carrier signal for fibre optic sensing applications. We demonstrated its capability using firstly an FBG sensing scheme then an interferometric sensing scheme. The differential nature of this type of interferometer means that for short lengths of $\mathrm{Hi}-\mathrm{Bi}$ fibre where a larger FSR is required the Hi-Bi fibre needs to be stretched enough to drive the interferometer over one fringe, although not demonstrated in this work it could prove to be a problem.

D. C. C. Norman, Y. Lai and D. J. Webb (Photonics Research Group, Aston University, Birmingham, B4 7ET, United Kingdom)

E-mail: d.j.webb@aston.ac.uk

\section{References}

1 YU, F. T. S., and YIN, S.: 'Fiber Optic Sensors', Marcel Dekker Inc 2002, pp. 295-480

2 Y. LAI.: 'Fibre-based devices for next generation photonics communication systems, Ph.D Thesis, Aston University, 2004

3 KERSEY, A. D., BERKOFF, T. A., and MOREY, W. W.: 'Fibre-optic Bragg grating strain sensor with drift-compensated high-resolution wavelength shift detection', Optics Letters, Vol. 18, No. 1, January 1993, pp. $72-74$

4 KERSEY, A. D., and DANDRIDGE, A.: 'Dual-wavelength approach to interferometric sensing', Proc. SPIE, Vol. 798, Fibre Optic Sensors 1987, pp. 176-181

5 NORMAN, D. C. C., WEBB, D. J., and PECHSTEDT, R. D.: 'Extended range interrogation of wavelength division multiplexed fibre Bragg grating sensors using arrayed waveguide grating', Electronics Letters, Vol. 39, Nov. 2003, pp. 1714-1716.

6 NORMAN, D. C. C., WEBB, D. J., and PECHSTEDT, R. D.:

'Interferometric sensor interrogation using an arrayed waveguide grating', Proc. SPIE, Vol. 5459, Optical Sensing, September 2004, pp. 101-109

7 PATTEN, R. A.: 'Michelson interferometer as a remote gauge', Applied Optics, Vol. 10, No. 12, 1971, pp. 2717-2721 